\title{
Interpolation of entire functions on regular sparse sets and $q$-Taylor series
}

\author{
par MiChaEL WELTER
}

RÉSUMÉ. Nous donnons une démonstration alternative d'un théorème de Ismail et Stanton et appliquons cela à des fonctions entières arithmétiques.

Abstract. We give a pure complex variable proof of a theorem by Ismail and Stanton and apply this result in the field of integer-valued entire functions. Our proof rests on a very general interpolation result for entire functions.

\section{Introduction}

In [4] (see also the references there) Ismail and Stanton established $q$ analogues of Taylor series expansions of entire functions, so-called $q$-Taylor series, and gave some applications of these. Their proofs depend heavily on the theory of basic hypergeometric functions.

In this note we will deduce one of their theorems from an interpolation formula which we will prove in section 2 . In section 3 we will give another application of the $q$-Taylor series in the field of the so-called integer-valued entire functions and give a first answer on a question asked by Ismail and Stanton in [4].

We start with some definitions and notations. Throughout this section let $q, a \in \mathbb{C} \backslash\{0\}$ with $|q| \neq 1$. We denote by $Q$ the maximum of $|q|$ and $\left|q^{-1}\right|$.

The $q$-shifted factorials are defined by

$$
(a ; q)_{0}:=1, \quad(a ; q)_{n}:=\left(1-a q^{n-1}\right)(a ; q)_{n-1} \text { for } n=1,2,3, \ldots
$$

We put

$$
z_{n}:=\frac{1}{2}\left(a q^{n}+a^{-1} q^{-n}\right)
$$

and

$$
\phi_{n}(z ; a):=\prod_{k=0}^{n-1}\left(1-2 a z q^{k}+a^{2} q^{2 k}\right) \in \mathbb{C}[z]
$$

This work was supported by the DFG (German Research Foundation). 
for $n=0,1,2,3, \ldots$. Finally we denote for an entire function $f$

$$
\sigma(f):=\limsup _{r \rightarrow \infty} \frac{\log |f|_{r}}{(\log r)^{2}}
$$

where as usual $|f|_{r}:=\max _{|z|=r}|f(z)|$. The theorem of Ismail and Stanton (Theorem 3.3 in [4]) states

Theorem 1.1. Let $f$ be an entire function with $\sigma(f)<1 /(2 \log Q)$. Then we have for all $z \in \mathbb{C}$

$$
f(z)=\sum_{n=0}^{\infty} q^{n} f_{n, \phi} \phi_{n}(z ; a)
$$

with

$$
f_{n, \phi}=\sum_{k=0}^{n} \frac{(-1)^{k} q^{k(k-1) / 2}\left(1-a^{2} q^{2 k}\right)}{(q ; q)_{k}(q ; q)_{n-k}\left(a^{2} q^{k} ; q\right)_{n+1}} f\left(z_{k}\right) .
$$

Remark. Ismail and Stanton state the theorem only for real $a, q$ with $0<a, q<1$.

\section{Interpolation of entire functions on regular sparse sets}

For subsets $X \subset \mathbb{C}$ we put $\psi_{X}(r)=\operatorname{card}\{x \in X|| x \mid \leq r\}$.

Definition. We call a subset $X \subset \mathbb{C}$ regular sparse, if $X$ is infinite, discrete and satisfies the following condition:

There exist $\theta \in] 1,+\infty[$ and $T \in \mathbb{R}$ such that

$$
\psi_{X}\left(r^{\theta}\right) \leq T \psi_{X}(r)+o\left(\psi_{X}(r)\right) \text { when } r \rightarrow+\infty .
$$

In [6] we studied entire functions $f$ that are integer-valued on regular sparse sets $X \subset \mathbb{Z}$. There we proved the following characterization of regular sparse sets (see [6], Lemma 1 ).

Lemma 2.1. Let $X$ be an infinite, discrete subset of $\mathbb{C}$. Then the following three statements are equivalent:

(i) $X$ is regular sparse.

(ii) For all $\theta \in] 1,+\infty\left[\right.$ there exists a $T \in \mathbb{R}$ such that $\psi_{X}\left(r^{\theta}\right) \leq T \psi_{X}(r)+$ $o\left(\psi_{X}(r)\right)$ when $r \rightarrow+\infty$.

(iii)

$$
\bar{\Lambda}(X):=\limsup _{r \rightarrow \infty} \frac{1}{\log r \psi_{X}(r)} \sum_{\substack{x \in X \backslash\{0\} \\ 1<|x| \leq r}} \log |x|<1 .
$$

Therefore it is useful to define

$$
T_{X}(\theta):=\limsup _{r \rightarrow+\infty} \frac{\psi_{X}\left(r^{\theta}\right)}{\psi_{X}(r)} \in[1,+\infty[
$$

and the main result of this section states as follows. 
Theorem 2.2. Let $X$ be a regular sparse subset of $\mathbb{C}$ and let $\left(x_{n}\right)_{n \in \mathbb{N}_{0}}$ be the sequence of all distinct elements of $X$ ordered by increasing modulus. Then we have for all entire functions $f$ with

$$
\limsup _{r \rightarrow+\infty} \frac{\log |f|_{r}}{\psi_{X}(r) \log r}<\sup _{\theta \in] 1,+\infty[} \frac{\theta-\bar{\Lambda}(X)}{\theta T_{X}(\theta)}=: \gamma_{0}
$$

the series expansion

$$
f(z)=\sum_{n=0}^{\infty} A_{X, n} P_{X, n}(z),
$$

where $P_{X, 0}(z):=1$ and $P_{X, n}(z):=\left(z-x_{n-1}\right) P_{X, n-1}(z)$ for all $n \geq 1$ and

$$
A_{X, n}=\sum_{k=0}^{n} \prod_{\substack{\nu=0 \\ \nu \neq k}}^{n}\left(x_{k}-x_{\nu}\right)^{-1} f\left(x_{k}\right)
$$

Therefore, every such $f$ is uniquely determined by its values on $X$.

Remark. In [6] we proved that the entire function $g$ which is defined by

$$
g(z):=\prod_{x \in X \backslash\{0\}}\left(1-\frac{z}{x}\right)
$$

has a growth bounded by

$$
\log |g|_{r} \leq(1-\underline{\Lambda}(X)) \psi_{X}(r) \log r+o\left(\psi_{X}(r) \log r\right)
$$

for all sufficiently large $r$, where

$$
\underline{\Lambda}(X):=\liminf _{r \rightarrow \infty} \frac{1}{\psi_{X}(r) \log r} \sum_{\substack{x \in X \backslash\{0\} \\ 1<|x| \leq r}} \log |x| .
$$

Before we prove the above theorem, we will deduce theorem 1.1 from it.

Proof of theorem 1.1. We set $X=\left\{z_{k} \mid k \in \mathbb{N}_{0}\right\}$. Then we have $\psi_{X}(r)=$ $\log r / \log Q+O(1)$ when $r \rightarrow+\infty, T_{X}(\theta)=\theta$ and $\bar{\Lambda}(X)=1 / 2$. Hence $(3)$ becomes $\sigma(f)<1 /(2 \log Q)$.

The polynomial $\phi_{n}(z ; a)$ is of degree $n$ in $z$ and has the property $\phi_{n}\left(z_{k} ; a\right)=0$ for $k=0, \ldots, n-1$. Hence $\phi_{n}(z ; a)=c_{n} P_{X, n}(z)$ with $c_{n}=(-1)^{n}(2 a)^{n} q^{n(n-1) / 2}$. 
We have

$$
\begin{aligned}
(q ; q)_{k} & =\prod_{\nu=1}^{k}\left(1-q^{\nu}\right)=\prod_{\nu=0}^{k-1}\left(1-q^{k-\nu}\right) \\
(q ; q)_{n-k} & =\prod_{\nu=1}^{n-k}\left(1-q^{\nu}\right)=\prod_{\nu=k+1}^{n}\left(1-q^{\nu-k}\right) \\
\frac{\left(a^{2} q^{k} ; q\right)_{n+1}}{\left(1-a^{2} q^{2 k}\right)} & =\left(1-a^{2} q^{2 k}\right)^{-1} \prod_{\nu=0}^{n}\left(1-a^{2} q^{k+\nu}\right) \\
& =\prod_{\nu=0}^{k-1}\left(1-a^{2} q^{k+\nu}\right) \prod_{\nu=k+1}^{n}\left(1-a^{2} q^{k+\nu}\right)
\end{aligned}
$$

and

$$
\begin{aligned}
& \left(1-q^{k-\nu}\right)\left(1-a^{2} q^{k+\nu}\right)=2 a q^{k}\left(z_{k}-z_{\nu}\right) \\
& \left(1-q^{\nu-k}\right)\left(1-a^{2} q^{k+\nu}\right)=-2 a q^{\nu}\left(z_{k}-z_{\nu}\right) .
\end{aligned}
$$

From this we deduce

(5)

$(q ; q)_{k}(q ; q)_{n-k} \frac{\left(a^{2} q^{k} ; q\right)_{n+1}}{\left(1-a^{2} q^{2 k}\right)}=(-1)^{n-k}(2 a)^{n} q^{n(n+1) / 2+k(k-1) / 2} \prod_{\substack{\nu=0 \\ \nu \neq k}}^{n}\left(z_{k}-z_{\nu}\right)$.

And therefore we get from (4)

$$
q^{n} f_{n, \phi} c_{n}=\sum_{k=0}^{n} \prod_{\substack{\nu=0 \\ \nu \neq k}}^{n}\left(z_{k}-z_{\nu}\right)^{-1} f\left(z_{k}\right)=A_{X, n}
$$

This proves Theorem 1.1

Proof of theorem 2.2. Without loss of generality we assume that $|x| \geq 1$ for all $x \in X$.

Let $n$ be a positive integer, which we assume to be sufficiently large. Let $r$ be a real with $r>\left|x_{n-1}\right|$. We will specify $r$ a little bit later in the proof. For every $z \in \mathbb{C}$ with $|z|<r$ we have (see e.g. Bundschuh [3])

$$
f(z)=\sum_{\nu=0}^{n-1} A_{X, \nu} P_{X, \nu}(z)+R_{X, n}(z)
$$

where

$$
A_{X, \nu}:=\frac{1}{2 \pi i} \int_{|\xi|=r} \frac{f(\xi) d \xi}{P_{X, \nu+1}(\xi)}
$$


and

$$
R_{X, n}(z):=\frac{P_{X, n}(z)}{2 \pi i} \int_{|\xi|=r} \frac{f(\xi) d \xi}{(\xi-z) P_{X, n}(\xi)} .
$$

Obviously (4) follows from (8) by Cauchy's integral formula.

To prove the theorem, it is therefore enough to prove that under the assumptions of Theorem 2.2 the reminder $R_{X, n}$ converges uniformly against the zero function on any compact subset of $\mathbb{C}$.

We suppose that we have $\log |f|_{r} \leq \gamma \psi_{X}(r) \log r$ with a constant $\gamma<\gamma_{0}$ for all sufficiently large $r$. Further we fix a $\theta \in] 1,+\infty[$ such that

$$
\gamma<\frac{\theta-\bar{\Lambda}(X)}{\theta T_{X}(\theta)}
$$

Let $\delta>0$ and $z \in \mathbb{C}$ with $|z| \leq \delta$.

For $\theta>1$, we have $2\left|x_{n}\right| \leq\left|x_{n}\right|^{\theta}=: r$ for all sufficiently large $n$. Therefore we can estimate

$$
\prod_{\nu=0}^{n-1}\left(1-\frac{\left|x_{\nu}\right|}{\left|x_{n}\right|^{\theta}}\right) \geq\left(\frac{1}{2}\right)^{n}=\exp \left(O\left(\psi_{X}(r)\right)\right) .
$$

The last equality follows from the fact that $\psi_{X}\left(\left|x_{n}\right|\right)=n+O(1)$ for all $n$. By Proposition 1 of [6] we know, that for regular sparse sets $X$ there are constants $c, \alpha>0$ such that $\log \left|x_{n}\right| \geq c n^{\alpha}$ for all $n$. Hence the limit

$$
C(\delta):=\lim _{n \rightarrow \infty} \prod_{\nu=0}^{n-1}\left(1+\frac{\delta}{\left|x_{\nu}\right|}\right)
$$

exists. This leads to

$$
\left|P_{X, n}(z)\right|=\left|\prod_{\nu=0}^{n-1}\left(z-x_{\nu}\right)\right| \leq C(\delta) \prod_{\nu=0}^{n-1}\left|x_{\nu}\right| \leq C \exp \left(\sum_{\substack{x \in X \\|x| \leq\left|x_{n}\right|}} \log |x|\right)
$$

and for all $\xi$ with $|\xi|=r$

$$
\left|P_{X, n}(\xi)\right| \geq \prod_{\nu=0}^{n-1}\left(\left|x_{n}\right|^{\theta}-\left|x_{\nu}\right|\right) \geq \exp \left(\theta \psi_{X}\left(\left|x_{n}\right|\right) \log \left|x_{n}\right|+O\left(\psi_{X}(r)\right)\right)
$$

Further we have if $n$ and therefore $r$ is sufficiently large

$$
\begin{aligned}
\log |f|_{r} & \leq \gamma \psi_{X}\left(\left|x_{n}\right|^{\theta}\right) \log \left|x_{n}\right|^{\theta} \\
& \leq \gamma T_{X}(\theta) \theta \psi_{X}\left(\left|x_{n}\right|\right) \log \left|x_{n}\right|+o\left(\psi_{X}\left(\left|x_{n}\right|\right) \log \left|x_{n}\right|\right) .
\end{aligned}
$$

Here we have again used the fact that the set $X$ is regular sparse. 
If we further assume, that $2 \delta<r$, then we get from (9)

$$
\begin{aligned}
\left|R_{X, n}(z)\right| \leq \exp \left(\left(\bar{\Lambda}(X)-\theta+\gamma \theta T_{X}(\theta)\right) \psi_{X}\left(\left|x_{n}\right|\right)\right. & \log \left|x_{n}\right| \\
& \left.+o\left(\psi_{X}\left(\left|x_{n}\right|\right) \log \left|x_{n}\right|\right)\right)
\end{aligned}
$$

which shows that $R_{X, n}(z)$ converges against zero when $n$ tends to infinity. Hence the theorem is proven.

\section{Application of Theorem 1.1 to integer-valued entire functions}

In this section we will give some statements about entire functions that are integer-valued on the sequence $z_{n}=\left(a q^{n}+a^{-1} q^{-n}\right) / 2$.

The following theorem is a corollary to Theorem 1 in [6], a general result on integer-valued entire functions on regular sparse sets $X \subset \mathbb{Z}$. From this theorem one easily deduces

Theorem 3.1. Let $a, q \in \mathbb{C} \backslash\{0\}$ with $|q| \neq 1$ such that $z_{n}:=\frac{1}{2}\left(a q^{n}+\right.$ $\left.a^{-1} q^{-n}\right) \in \mathbb{Z}$ for every $n \in \mathbb{N}_{0}$, and let $f$ be an entire function such that $f\left(z_{n}\right) \in \mathbb{Z}$ for every $n \in \mathbb{N}_{0}$ and

$$
\log |f|_{r} \leq \gamma \frac{(\log r)^{2}}{\log |q|}, \gamma<0.0225
$$

for all sufficiently large $r$. Then $f$ is a polynomial function.

Remark. The case $a= \pm 1$ was essentially treated by Bézivin in [1,2]. By using an interpolation series method he obtained a better upper bound for $\gamma$ than that in the above theorem. The sequence $\left(z_{n}\right)$ is the solution of the linear difference equation $u_{n+1}=\left(q+q^{-1}\right) u_{n}-u_{n-1}$ with the initial values $u_{0}=\left(a+a^{-1}\right) / 2$ and $a_{1}=\left(a q+a^{-1} q^{-1}\right) / 2$. Hence the condition $z_{n} \in \mathbb{Z}$ for all $n \in \mathbb{N}_{0}$ is obviously satisfied if the three numbers

$$
\frac{a+a^{-1}}{2}, \frac{a q+a^{-1} q^{-1}}{2}, q+q^{-1}
$$

are rational integers. Therefore the theorem above covers not only the case $a= \pm 1$ and we get some new applications with $a, q$ both lying in the same real quadratic number field.

From the $q$-Taylor theorem 1.1, we can deduce the following result, which covers another case.

Theorem 3.2. Let $\mathbb{K}$ be $\mathbb{Q}$ or an imaginary-quadratic number field and $O_{\mathbb{K}}$ be its ring of integers. Further let $a, q \in O_{\mathbb{K}} \backslash\{0\}$ with $|q|>1$ and $a^{2} \notin\left\{q^{-\nu} \mid \nu \in \mathbb{N}\right\}$. If $f$ is an entire function satisfying $f\left(z_{n}\right) \in O_{\mathbb{K}}$ for all $n \in \mathbb{N}_{0}$ and

$$
\log |f|_{r} \leq \gamma \frac{(\log r)^{2}}{\log |q|}, \text { where } \gamma<1 / 10
$$

for all sufficiently large $r$, then $f$ is a polynomial function. 
Proof. If $\Phi_{d}$ denotes the $d$-th cyclotomic polynomial, then we have for all $n \in \mathbb{N}$ and $k \in\{0, \ldots, n\}$ (see Lang [5], p. 279f.)

$$
\frac{(q ; q)_{n}}{(q ; q)_{k}(q ; q)_{n-k}}=\prod_{d=1}^{n} \Phi_{d}(q)^{\left[\frac{n}{d}\right]-\left[\frac{k}{d}\right]-\left[\frac{n-k}{d}\right]} \in \mathbb{Z}[q] .
$$

Obviously we have

$$
\frac{\prod_{\nu=1}^{2 n-1}\left(1-a^{2} q^{\nu}\right)}{\prod_{\nu=0}^{k-1}\left(1-a^{2} q^{k+\nu}\right) \prod_{\nu=k+1}^{n}\left(1-a^{2} q^{k+\nu}\right)} \in \mathbb{Z}[a, q] .
$$

Hence, if we put $D_{n}:=(q ; q)_{n} \prod_{\nu=1}^{2 n-1}\left(1-a^{2} q^{\nu}\right) \neq 0$, it follows from (5) and (6) that $D_{n} A_{X, n} \in \mathbb{Z}[a, q]$ for all $n \in \mathbb{N}_{0}$. Therefore $\left|D_{n} A_{X, n}\right| \geq 1$, if $D_{n} A_{X, n}$ is not equal to zero.

On the other hand, we find by (8) like in the proof of Theorem 2.2, again with $r:=\left|z_{n}\right|^{\theta}$

$$
\left|A_{X, n}\right| \leq \exp \left(\left(\gamma \theta^{2}-\theta\right) n^{2} \log |q|+o\left(n^{2}\right)\right)
$$

and

$$
\left|D_{n}\right| \leq|q|^{\frac{n(n+1)}{2}+\frac{(2 n-1) 2 n}{2}} \prod_{\nu=1}^{n}\left(1+|q|^{-\nu}\right) \prod_{\nu=1}^{2 n-1}\left(1+\left|a^{2}\right||q|^{-\nu}\right) .
$$

Obviously the two infinite products

$$
\prod_{\nu=1}^{\infty}\left(1+|q|^{-\nu}\right)
$$

and

$$
\prod_{\nu=1}^{\infty}\left(1+\left|a^{2}\right||q|^{-\nu}\right)
$$

converge, and therefore we get

$$
\left|D_{n} A_{X, n}\right| \leq \exp \left(\left(\gamma \theta^{2}-\theta+5 / 2\right) n^{2} \log |q|+o\left(n^{2}\right)\right) .
$$

We now chose $\theta=1 /(2 \gamma)$. If $\gamma<1 / 10$ then the upper bound of $\left|D_{n} A_{X, n}\right|$ is less than 1 for all sufficiently large $n$. Hence $D_{n} A_{X, n}=0$ for this $n$. For we know that $D_{n}$ is not zero, this implies that $A_{X, n}$ vanishes for all sufficiently large $n$. This proves the theorem.

\section{References}

[1] J.-P. BÉZIVIN, Sur les points où une fonction analytique prend des valeurs entières. Ann. Inst. Fourier 40 (1990), 785-809.

[2] J.-P. BÉzIvin, Fonctions entières prenant des valeurs entières ainsi que ses dérivées sur des suites recurrentes binaires. Manuscripta math. 70 (1991), 325-338.

[3] P. Bundschum, Arithmetische Eigenschaften ganzer Funktionen mehrerer Variablen. J. reine angew. Math. 313 (1980), 116-132. 
[4] M. E. H. Ismail, D. Stanton, $q$-Taylor theorems, polynomial expansions, and interpolation of entire functions. Journal of Approximation Theory 123 (2003), 125-146.

[5] S. LANG, Algebra. 3rd edition, Addison-Wesley (1993).

[6] M. WELTER, Ensembles régulièrement lacunaires d'entiers et fonctions entières arithmétiques. J. Number Th. 109 (2004), 163-181.

Michael WELTER

Mathematisches Institut der Universität Bonn

Beringstr. 4

53115 Bonn, Allemagne

E-mail : welter@math.uni-bonn.de 